\title{
Strong effects of weak localization in charge-density-wave/normal-metal hybrids
}

\author{
Mark I. Visscher, B. Rejaei, and Gerrit E. W. Bauer \\ Theoretical Physics Group, Department of Applied Physics and Delft Institute of Microelectronics and Submicron Technology, \\ Delft University of Technology, Lorentzweg 1, 2628 CJ Delft, The Netherlands
}

(Received 26 April 2000)

\begin{abstract}
Collective transport through a multichannel disordered conductor in contact with charge-density-wave electrodes is theoretically investigated. The statistical distribution function of the threshold potential for chargedensity-wave sliding is calculated by random matrix theory. In the diffusive regime weak localization has a strong effect on the sliding motion.
\end{abstract}

Wave (Anderson) localization is an important concept of the quantum theory of electron transport. It is well established by now that a random disorder potential may cause bound states with an exponential decay governed by the localization length. When this localization length is large compared to other length scales in the system, the corrections to semiclassical transport are small, and the term "weak localization" is appropriate. Weak localization is well understood for normal Fermi liquids. However, localization in heterostructures like superconducting metal/normal metal $(\mathrm{S} / \mathrm{N})$, or Josephson (S/N/S) junctions is still actively investigated, experimentally and theoretically. For example, the electronhole coherence induced in a normal metal by a superconducting reservoir significantly modifies the weak localization correction to the electron transport through the hybrid structure. ${ }^{1}$

A charge-density wave (CDW) is a collective state similar to BCS superconductors $(\mathrm{S}){ }^{2}$ For instance, $\mathrm{CDW}$ reservoirs induce a proximity effect in normal metals $(\mathrm{N})$ analogous to that in N/S junctions when the interface is parallel to the CD wave fronts (normal to the chains). In such a geometry the phase coupling through a defect-free CDW/N/CDW junction supports a sliding CDW mode. ${ }^{3}$ A process analogous to Andreev scattering at an N/CDW interface has been predicted. ${ }^{4}$ Advances in thin film growth and patterning of CDW compounds ${ }^{5}$ enables the study of CDW transport in various geometries on a mesoscopic scale. ${ }^{6,7}$ Quantum oscillations have been identified in the magnetoresistance of the CDW compound $\mathrm{NbSe}_{3}$ in the presence of columnar defects. ${ }^{8}$ Electrical contacts that inject the current parallel to the CDW chains allow detailed studies of the subgap conductance. ${ }^{9}$

In this paper we report characteristic effects of weak localization on CDW sliding through a disordered conductor. In contrast to the extensive literature on semiconductor- or superconductor hybrid structures, ${ }^{1}$ this topic has, to the best of our knowledge, not yet been investigated. The statistical properties of diffuse conductors, including the weaklocalization correction to the average conductance, are well described by random matrix theory. ${ }^{10}$ We calculate the statistical properties of the pinning energy in the presence and absence of time-reversal symmetry by averaging over random transfer matrices. We find that the pinning strength is much more sensitive to the localization-enhanced backward scattering than the normal conductance. Weak localization is destroyed when time reversal-symmetry is broken by a magnetic field, ${ }^{11}$ leading to a substantial negative magnetoresistance.

We consider a disordered metal with length $L$ and width $W$ sandwiched between two CDW reservoirs consisting of $N$ half-infinite chains (Fig. 1). The CDW state is characterized by a complex order parameter $\Delta \exp (i \chi)$, where the amplitude $2 \Delta$ is the gap in the electronic spectrum and its phase $\chi$ denotes the position of the CDW relative to the crystal lattice. The amplitude of the order parameter $\Delta$ is taken to be equal on all chains and constant up to the interfaces. In the limit of large correlation lengths $\xi=\hbar v_{F} / \Delta \gg L$, the Coulomb interaction between the chains and the phase coherence over the region allows us to define a single CDW phase $\chi$ for the whole structure. For simplicity the material parameters of the CDW and the normal metal are taken to be matched at the interface. This corresponds to a normal metallic island fabricated by ion damage ${ }^{8}$ or selective abrasion ${ }^{12}$ of the CDW material. Mismatch between a CDW and, e.g., a true two-dimensional (2D) or 3D metal or imperfect interfaces causes additional scattering, which, in principle, can be incorporated into our formalism.

Many semiconducting CDW materials are characterized by a nearly one-dimensional electron band structure. On the other hand, there are also CDW materials with substantial transverse dispersion, like $\mathrm{NbSe}_{3}$. For the latter systems, and in the diffusive $(L \gg l)$ and quasi-one-dimensional $(L \gg W)$

(a)

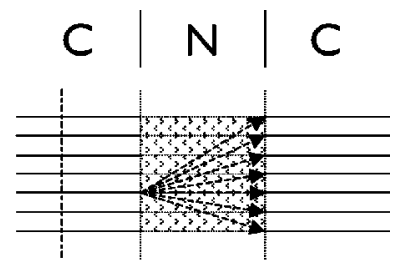

$\chi$

(b)

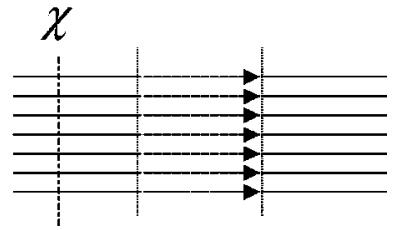

FIG. 1. Schematic pictures of a disordered metal in contact with CDW electrodes in the (a) isotropic and (b) anisotropic limits. $\chi$ denotes the position of the $\mathrm{CD}$ wave fronts. 
limits, an electron flux from one chain is distributed evenly among the outgoing chains. These two "anisotropic" and "isotropic" limiting cases (see Fig. 1) are treated below separately. Interpolation between these two extremes seems reasonable.

In a Wannier site representation with respect to individual chains, equilibrium properties of CDW's can be described by the retarded and advanced quasiclassical Green functions $\left(g_{\alpha \beta}\right)_{i j}^{R, A}(x, \epsilon)$, where $\alpha, \beta=\{R, L\}$, and $i, j=\{1, \ldots, N\} .{ }^{13}$ The subscripts $\{R, L\}$ refer to the right and left moving electrons on the linearized branches of the electronic spectrum, and $i, j$ are the chain indices. The $2 N \times 2 N$ matrix Green functions left $(-)$ and right $(+)$ of the scattering region are related by the boundary condition

$$
\mathbf{M}^{\dagger} \mathbf{g}_{+}=\mathbf{g}_{-} \mathbf{M}^{\dagger},
$$

where $\mathbf{M}$ is the $2 N \times 2 N$ transfer matrix of the disordered region that satisfies the condition $\mathbf{M}^{\dagger} \boldsymbol{\Sigma}_{3} \mathbf{M} \boldsymbol{\Sigma}_{3}=1$ in order to ensure current conservation, and $\mathbf{M}^{*}=\boldsymbol{\Sigma}_{1} \mathbf{M} \boldsymbol{\Sigma}_{1}$ in the presence of time-reversal symmetry. Here $\boldsymbol{\Sigma}_{1}$ and $\boldsymbol{\Sigma}_{3}$ are defined as

$$
\Sigma_{1}=\left(\begin{array}{ll}
0 & 1 \\
1 & 0
\end{array}\right), \quad \Sigma_{3}=\left(\begin{array}{cc}
1 & 0 \\
0 & -1
\end{array}\right) .
$$

Additional scattering at the interfaces mentioned above can be incorporated by substituting $\mathbf{M} \rightarrow \mathbf{M}_{R} \mathbf{M} \mathbf{M}_{L}$, where $\mathbf{M}_{R, L}$ are the transfer matrices of the right and left interfaces, respectively. The transfer matrix can be decomposed as ${ }^{11}$

$$
\mathbf{M}=\left(\begin{array}{ll}
\boldsymbol{\alpha}^{(1)} & \mathbf{0} \\
\mathbf{0} & \boldsymbol{\alpha}^{(3)}
\end{array}\right)\left(\begin{array}{cc}
(\mathbf{1}+\boldsymbol{\lambda})^{1 / 2} & \boldsymbol{\lambda}^{1 / 2} \\
\boldsymbol{\lambda}^{1 / 2} & (\mathbf{1}+\boldsymbol{\lambda})^{1 / 2}
\end{array}\right)\left(\begin{array}{ll}
\boldsymbol{\alpha}^{(2)} & \mathbf{0} \\
\mathbf{0} & \boldsymbol{\alpha}^{(4)}
\end{array}\right),
$$

where $\boldsymbol{\alpha}^{(i)}$ with $i=\{1,2,3,4\}$ are $N \times N$ unitary matrices, and $\boldsymbol{\lambda}$ is a real diagonal matrix with elements $0<\left\{\lambda_{1}, \ldots, \lambda_{N}\right\}$ $<\infty$, frequently referred to as eigenparameters of M. In the presence of time-reversal symmetry, $\boldsymbol{\alpha}^{(1) *}=\boldsymbol{\alpha}^{(3)}$, and $\boldsymbol{\alpha}^{(2) *}=\boldsymbol{\alpha}^{(4)}$.

Backscattering represented by the nondiagonal elements of $\mathbf{M}$ pins the $\mathrm{CDW}$ condensate, i.e., no current flows in linear response. By forcing the system out of the equilibrium position $\chi_{0}$, a chemical potential difference between the left and right side arises. The threshold potential $\mu_{T}$ at which the condensate starts to slide corresponds to the maximum value of the chemical potential difference as a function of the condensate position $\chi$ :

$$
\mu_{T} / 2 \equiv \max \left[\mu_{+}(\chi)-\mu_{-}(\chi)\right] .
$$

$\left[\mu_{+}-\mu_{-}\right](\chi)$ can be identified as the periodic pinning potential. In terms of the retarded and advanced Green functions, the chemical potentials $\mu_{ \pm}$of left and right CDW's read

$$
\mu_{ \pm}=-\frac{1}{8 N} \int d \epsilon \tanh \left(\frac{\epsilon}{2 k_{B} T}\right) \operatorname{Tr} \boldsymbol{\Sigma}_{3}\left(\mathbf{g}_{ \pm}^{R}-\mathbf{g}_{ \pm}^{A}\right) .
$$

The pinning potential is expressed in terms of the transfer matrix elements via the Green functions $\mathbf{g}_{ \pm}^{R, A}$, which can be obtained from their equation of motion ${ }^{13}$ and the boundary condition Eq. (1). In the energy integral of Eq. (5), the con- tinuum contribution to the energy integration dominates the bound states contribution when $\epsilon_{F} \gg \Delta$. In this limit the bound states on the island may be disregarded, and we arrive via Eq. (4) at the simple expression:

$$
\mu_{T}=\frac{\Delta}{N \gamma}\left|\operatorname{Tr}\left(\mathbf{r}^{\prime}-\mathbf{r}^{\dagger}\right)\right|,
$$

where $\mathbf{r}^{\prime}=\boldsymbol{\alpha}^{(1)}(\boldsymbol{\lambda} /(\mathbf{1}+\boldsymbol{\lambda})) \boldsymbol{\alpha}^{(3) \dagger}$ and $\mathbf{r}=-\boldsymbol{\alpha}^{(4)}(\boldsymbol{\lambda} /(\mathbf{1}+\boldsymbol{\lambda})) \boldsymbol{\alpha}^{(2) \dagger}$ are the $(N \times N)$ reflection matrices of the disordered region, and $\gamma \ll 1$ is the dimensionless electron-phonon coupling constant. ${ }^{14}$ The threshold potential (6) is proportional to a factor containing CDW material parameters and a "form factor" depending on transfer matrix elements of the disordered region. In contrast to the reflection probability $\operatorname{Tr} \mathbf{r}^{\dagger} \mathbf{r}, \mu_{T}$ is not invariant under a transformation $\mathbf{r} \rightarrow \mathbf{u r v}$, where $\mathbf{u}, \mathbf{v}$ are arbitrary unitary matrices. This is a direct consequence of the broken translational invariance of CDW's in the presence of defects. Naturally, the threshold is positive definite. By extending it symmetrically to negative threshold values the distribution function can be constructed from the averages of all even moments.

The theory of random matrices is first employed for the isotropic limit described above. The ensemble average of a quantity $f(\mathbf{M})$ of a collection of random conductors with length $L$ is defined as

$$
\langle f\rangle=\int f(\mathbf{M}) p_{L}(\lambda) d \mu(\mathbf{M}),
$$

where $d \mu(\mathbf{M})$ is the invariant or Haar measure, and $p_{L}(\lambda)$ is the isotropic probability density. ${ }^{11}$ In terms of the parametrization in Eq. (3), the measure is explicitly given by

$$
d \mu(\mathbf{M})=J_{\beta}(\boldsymbol{\lambda}) d^{N} \lambda \prod_{i=1}^{2 \beta} d \mu\left(\boldsymbol{\alpha}^{(i)}\right),
$$

where the Jacobian

$$
J_{\beta}(\boldsymbol{\lambda})=\prod_{a<b}\left|\lambda_{a}-\lambda_{b}\right|^{\beta},
$$

and $d \mu\left(\boldsymbol{\alpha}^{(i)}\right)$ are the invariant measures of the unitary group. The symmetry index $\beta=1$ describes the orthogonal ensemble in the presence of time-reversal symmetry, and the unitary ensemble $\beta=2$ implies broken time-reversal symmetry. The isotropic distribution function $P(\boldsymbol{\lambda}, L)=p_{L}(\boldsymbol{\lambda}) J(\boldsymbol{\lambda})$ satisfies an $N$-dimensional Fokker-Planck equation with the mean-free path $l$ as single parameter. ${ }^{11}$ From the calculated average in Eq. (7) it is possible to extract the distribution $\mathcal{P}\left(\mu_{T}\right)$ of the threshold indirectly via

$$
\langle f\rangle=\int_{0}^{\infty} d \mu_{T} f\left(\mu_{T}\right) \mathcal{P}\left(\mu_{T}\right) .
$$

In the following \langle\rangle$_{\alpha}$ and \langle\rangle$_{\lambda}$ denote the average over unitary matrices and eigenparameters, respectively.

We start by considering the ensemble averages of the $2 n$th powers of Eq. (6). All even moments $\left\langle\mu_{T}^{2 n}\right\rangle^{(\beta)}$ can be generated from the quantity

$$
\left\langle e^{\eta \operatorname{Tr}\left(\mathbf{r}^{\prime}-\mathbf{r}^{\dagger}\right) \operatorname{Tr}\left(\mathbf{r}^{\prime} 亡 \mathbf{r}\right)}\right\rangle^{(\beta)},
$$


by a Taylor expansion in $\eta$. It is convenient to write this as a Gaussian integral over the generating variables $\varphi$ and $\varphi^{*}$,

$$
\begin{aligned}
& e^{\eta \operatorname{Tr}\left(\mathbf{r}^{\prime}-\mathbf{r}^{\dagger}\right) \operatorname{Tr}\left(\mathbf{r}^{\prime} \leftarrow \mathbf{r}\right)}=\frac{1}{2 \pi i \eta} \int d \varphi d \varphi^{*} \\
& \left.\times e^{-|\varphi|^{2} / \eta+\varphi \operatorname{Tr}\left(\mathbf{r}^{\prime} \dagger\right.} \mathbf{r}\right)+\varphi^{*} \operatorname{Tr}\left(\mathbf{r}^{\prime}-\mathbf{r}^{\dagger}\right) .
\end{aligned}
$$

In this form we can average $\mathbf{r}$ and $\mathbf{r}^{\prime}$ separately over the unitary matrices, since the number of normal and Hermitian conjugated matrices must be equal in order to give nonzero contributions to the average. ${ }^{15}$ Equation (11) may therefore be rewritten as

$$
\left\langle\frac{1}{\eta} \int_{0}^{\infty} d|\varphi|^{2} e^{-|\varphi|^{2} / \eta}\left[\sum_{n=0}^{\infty} \frac{1}{(n !)^{2}}|\varphi|^{2 n}\left\langle\left(\operatorname{Tr} \mathbf{r}^{\dagger} \operatorname{Tr} \mathbf{r}\right)^{n}\right\rangle_{\alpha}\right]^{2}\right\rangle_{\lambda}^{(\beta)} .
$$

The first order term in the summation $(n=1)$ can be evaluated exactly;

$$
\left\langle\operatorname{Tr} \mathbf{r} \operatorname{Tr} \mathbf{r}^{\dagger}\right\rangle^{(\beta)}=\frac{2}{\beta N+2-\beta}\langle R\rangle_{\lambda}^{(\beta)},
$$

where we used $R=\operatorname{Tr} \mathbf{r}^{\dagger} \mathbf{r}=\operatorname{Tr} \boldsymbol{\lambda}(\mathbf{1}+\boldsymbol{\lambda})^{-1}$. This result agrees with the microscopic calculations of Dorokhov ${ }^{16}$ for the statistical distribution of the reflection matrix of a thin wire with Gaussian white-noise potentials and interchain hopping. From this relation we learn that the threshold potential is determined by the (diagonal) reflection probabilities $\left\langle\left|r_{i i}\right|^{2}\right\rangle^{(\beta)}$ at each chain. The $\beta$ dependent prefactor reflects the enhancement of backscattering by a factor 2 , which is suppressed when time-reversal symmetry is broken. ${ }^{11}$ It is instructive to compare this result with the reflectance of the disordered region $\left\langle\operatorname{Tr} \mathbf{r}^{\dagger} \mathbf{r}\right\rangle^{(\beta)}=\langle R\rangle_{\lambda}^{(\beta)}$, which differs only by the absence of the prefactor. The localization correction to the normal resistance recognized by its $\beta$ dependence is called weak because it vanishes to lowest order in $N$. In contrast, the pinning due to $\mathrm{CD}$ modulations induced by the reservoirs is strongly affected. In the limit $N \gg 1$, the (squared) pinning strength is doubled $\left\langle\mu_{T}^{2}\right\rangle^{(1)}=2\left\langle\mu_{T}^{2}\right\rangle^{(2)}$ by the weak-localization enhanced backscattering at each chain.

For the $n$th power $\left\langle\left(\operatorname{Tr} \mathbf{r}^{\dagger} \operatorname{Tr} \mathbf{r}\right)^{n}\right\rangle_{\alpha}$ we found analytical expressions only for large $N$. In the Gaussian limit ${ }^{17}$

$$
\begin{gathered}
\left\langle\left(\operatorname{Tr} \mathbf{r}^{\dagger} \operatorname{Tr} \mathbf{r}\right)^{n}\right\rangle_{\alpha}=n !\left(\frac{2}{\beta}\right)^{n}\left(\frac{\langle R\rangle_{\lambda}^{(\beta)}}{N}\right)^{n}, \\
\left.\left\langle e^{\eta \operatorname{Tr}\left(\mathbf{r}^{\prime}-\mathbf{r}^{\dagger}\right) \operatorname{Tr}\left(\mathbf{r}^{\prime} \dagger\right.} \mathbf{r}\right)\right\rangle^{(\beta)}=\left[1-\frac{4 \eta}{\beta}\left(\frac{s}{s+1}\right)\right]^{-1}
\end{gathered}
$$

to leading order $O\left(N^{0}\right)$. Here, the average over the eigenparameters $\boldsymbol{\lambda}$ has been performed to leading order in $N$ using the classical solution $\left\langle R^{n}\right\rangle_{\lambda}^{(\beta)}=(N s /(s+1))^{n}$ with $s=L / l{ }^{10}$ Comparing the coefficients with powers of $\eta$ on both sides, we finally arrive at a Wigner-like distribution function

$$
\mathcal{P}_{I}^{(\beta)}\left(\mu_{T}\right)=\frac{2 \beta \mu_{T}}{\left\langle\mu_{T}^{2}\right\rangle} e^{-\beta \mu_{T}^{2} /\left\langle\mu_{T}^{2}\right\rangle}
$$

for $0<\mu_{T}<\infty$, and $\left\langle\mu_{T}^{2}\right\rangle=2(\Delta / N \gamma)^{2}(s / s+1)$. The mean value and the standard deviation $\sigma$ are easily calculated as $\left\langle\mu_{T}\right\rangle^{(\beta)}=\Delta / N \gamma \sqrt{\pi s / \beta(s+1)}$ and $\sigma=\sqrt{3} \approx 1.732$. By breaking time-reversal symmetry, a magnetic field destroys the weak-localization enhanced backscattering, thus reducing the average threshold potential by a factor of $1 / \sqrt{2} \approx 0.71$ even though the normal resistance remains unchanged.

We now examine the anisotropic limit in which interchain scattering is absent. Time-reversal symmetry cannot be broken by a magnetic field, so we can restrict the discussion to the $\beta=1$ ensemble. The distribution of the unitary matrices is not random but sharply centered around the unit matrix. Therefore we take the unitary matrices to be diagonal. For a weak impurity $\lambda \ll 1$ located on a single chain $N=1$, we obtain in the ballistic limit $(s \ll 1)$ :

$$
\mathcal{P}_{A}\left(\mu_{T}\right)=\frac{2}{\sqrt{\pi\left\langle\mu_{T}^{2}\right\rangle}} e^{-\mu_{T}^{2} /\left\langle\mu_{T}^{2}\right\rangle}, \quad N=1
$$

with $\left\langle\mu_{T}^{2}\right\rangle=(2 \Delta / \gamma)^{2} s$ The distribution of pinning energies of a defect localized to a single chain satisfies a "half", Gaussian distribution, with an average $\left\langle\mu_{T}\right\rangle=(2 \Delta / \gamma) \sqrt{s / \pi}$. In the large- $N$ expansion the probability for zero threshold vanishes according to

$$
\mathcal{P}_{A}\left(\mu_{T}\right)=\frac{2 \mu_{T}}{\left\langle\mu_{T}^{2}\right\rangle} e^{-\mu_{T}^{2}\left\langle\mu_{T}^{2}\right\rangle}, \quad N \gg 1,
$$

where $\left\langle\mu_{T}^{2}\right\rangle=2 / N(\Delta / \gamma)^{2} s /(s+1)$ for arbitrary lengths $s$.

A negative magnetoresistance, which is caused by the reduced threshold potential in high magnetic fields, ${ }^{8}$ has been observed in $\mathrm{NbSe}_{3}$ in the presence of columnar defects. In semiconductor CDW materials like $\mathrm{Rb}_{0.3} \mathrm{MnO}_{3}$ or $\mathrm{TaS}_{3}$, the effect has, to our knowledge, not been reported. These observations are consistent with our findings, since the anisotropy of the normal state conductance of $\mathrm{NbSe}_{3}$ is low, in contrast to the fully gapped, strongly anisotropic CDW materials.

Our results also support previous work related to the periodicity of the Aharonov-Bohm oscillations. ${ }^{18}$ As shown here, the average threshold potential of a multichannel disordered conductor is sensitive to weak-localization trajectories. In the weak pinning limit, the effective threshold potential of many uncorrelated columnar defects as modelled by metallic islands is given by its averaged value. Therefore, we can argue that the latter is periodic in $h / 2 e$, since this component reflects the contribution from time-reversed paths. Finally, we point out that in the presence of many uncorrelated metallic islands the distribution of thresholds smears out the threshold singularity in the current-voltage characteristics and determines the functional behavior of the nonlinear conductance.

We conclude by summarizing our results. We present expressions for the threshold potential for collective CDW current through a multichannel disordered conductor in terms of its reflection matrices. By means of random matrix theory we calculate the statistical distribution of the pinning strength with and without time-reversal symmetry. We find that localization, which is weak on the normal conductance, has a 
strong effect on the threshold potential. The breaking of time-reversal symmetry by a magnetic field reduces the threshold field, depending on the isotropy of the disordered conductor. The magnitude of the reduction ranges from zero in the anisotropic limit to $1 / \sqrt{2}$ in the isotropic limit.
It is a pleasure to thank Carlo Beenakker for valuable discussions. This work is part of the research program for the "Stichting voor Fundamenteel Onderzoek der Materie", (FOM). We acknowledge support from the NEDO joint research program (NTDP-98).
${ }^{1}$ Transport Phenomena in Mesoscopic Systems, edited by H. Fukuyama and T. Ando (Springer Verlag, Berlin, 1992); Mesoscopic Phenomena in Solids, edited by B.L. Altshuler, P.A. Lee, and R.A. Webb (North-Holland, Amsterdam, 1991).

${ }^{2}$ G. Grüner, Density Waves in Solids (Addison-Wesley, Reading, MA, 1994); Charge Density Waves in Solids, edited by L. Gor'kov and G. Grüner (Elsevier Science, Amsterdam, 1989).

${ }^{3}$ M.I. Visscher and G.E.W. Bauer, Phys. Rev. B 54, 8487 (1996).

${ }^{4}$ B. Rejaei and G.E.W. Bauer, Phys. Rev. B 54, 8487 (1996).

${ }^{5}$ H.S.J. van der Zant, O.C. Mantel, C. Dekker, J.E. Mooij, and C. Traeholt, Appl. Phys. Lett. 68, 3823 (1996).

${ }^{6}$ Yu.I. Latyshev, B. Pannetier, and P. Monceau, Eur. Phys. J. B 3, 421 (1998).

${ }^{7}$ N. Markovic, M.A.H. Dohmen, and H.S.J. van der Zant, Phys. Rev. Lett. 84, 534 (2000); O.C. Mantel, F. Chalin, C. Dekker, H.S.J. van der Zant, Yu.I. Latyshev, P. Pannetier, and P. Monceau, ibid. 84, 538 (2000).

${ }^{8}$ Yu.I. Latyshev, O. Laborde, P. Monceau, and S. Klaumünzer, Phys. Rev. Lett. 78, 919 (1997).

${ }^{9}$ A.A. Sinchenko, Yu.I. Latyshev, S.G. Zybtsev, and I.G. Gorlova,
Zh. Eksp. Teor. Fiz 113, 1830 (1998) [J. Exp. Theor. Phys. 86, 1001 (1998)]; A.A. Sinchenko, Yu.I. Latyshev, S.G. Zybtsev, I.G. Gorlova, and P. Monceau, Phys. Rev. B 60, 4624 (1999).

${ }^{10}$ C.W.J. Beenakker, Rev. Mod. Phys. 69, 731 (1997); A.D. Stone, P.A. Mello, K.A. Muttalib, and J.-L. Pichard, in Mesoscopic Phenomena in Solids, edited by B.L. Altshuler, P.A. Lee, and R.A. Webb (North-Holland, Amsterdam, 1991).

${ }^{11}$ P.A. Mello and A.D. Stone, Phys. Rev. B 44, 3559 (1991).

${ }^{12}$ H.S.J. van der Zant (private communication).

${ }^{13}$ S.N. Artemenko, Zh. Eksp. Teor. Fiz 111, 1494 (1997) [Sov. Phys. JETP 84, 823 (1997)].

${ }^{14}$ For simplicity we have taken $\mathbf{M}$ to be energy independent. $\mathbf{M}$ is then evaluated for particles at the Fermi surface. This is a good approximation when the dwelling time of the particles on the island is small compared to $\hbar / \Delta$.

${ }^{15}$ P.A. Mello, J. Phys. A 23, 4061 (1990).

${ }^{16}$ O.N. Dorokhov, Phys. Rev. B 37, 10526 (1988).

${ }^{17}$ P.W. Brouwer, Ph.D thesis, Leiden University, 1997.

${ }^{18}$ M.I. Visscher and B. Rejaei, Europhys. Lett. 43, 617 (1998). 